\title{
Tumor eradication by immunotherapy with biodegradable PLGA microspheres-an alternative to incomplete Freund's adjuvant
}

\author{
Marc Mueller ${ }^{1}$, Eva Schlosser ${ }^{1}$, Bruno Gander ${ }^{2}$ and Marcus Groettrup ${ }^{1,3}$ \\ ${ }^{1}$ Division of Immunology, Department of Biology, Universität Konstanz, Konstanz, Germany \\ 'Institute of Pharmaceutical Sciences, Swiss Federal Institute of Technology (ETH) Zurich, Zurich, Switzerland \\ ${ }^{3}$ Biotechnology Institute Thurgau, Kreuzlingen, Switzerland
}

\begin{abstract}
In experimental tumor immunotherapy, incomplete Freund's adjuvant (IFA) has been considered as the "gold standard" for Tcell vaccination in mice and humans in spite of its considerable adverse effects. Recently, we succeeded in eliciting strong CTL responses in mice after vaccination with biodegradable poly(D,L-lactide-co-glycolide) (PLGA) microspheres (MS). In our study, we compared the immune response to IFA and PLGA-MS containing ovalbumin (OVA) and CpG-oligodeoxynucleotide (MS-OVA/CpG) or we used a mixture of MS-OVA/CpG and MS-polyl:C. A single vaccination with MS-OVA/CpG elicited longlasting titers of IgG1 and IgG2a, but only low IgE titers, and also the T-cell response was biased toward $\mathrm{Th}_{1}$ differentiation. Antigen presentation to $\mathrm{CD}_{4}^{+}$and $\mathrm{CDB}^{+}$cells and activation of a cytotoxic T-cell response in mice vaccinated with PLGA-MS and IFA lasted for over 3 weeks. Preconditioning of the injection site with TNF- $\alpha$ and heterologous prime-boost regimen further enhanced the cytotoxic response. PLGA-MS were as efficient or superior to IFA in eradication of preexisting tumors and suppression of lung metastases. Taken together, PLGA-MS are well-defined, biodegradable and clinically compatible antigen carrier systems that compare favorably with IFA in their efficacy of tumor immunotherapy in mouse models and hence deserve to be tested for their effectiveness against human malignant diseases.
\end{abstract}

The recent identifications of tumor antigens in numerous types of cancers and of ligands of pattern-recognition receptors are important prerequisites to elicit strong CTL responses against tumor cells by immunotherapy. Pattern molecules should be directed to their cognate receptors localized either on the cell surface, in the endosomal membrane or in the cytoplasm. Strong adjuvants like cytosine-phosphorothioate-guanine oligodeoxynucleotides $(\mathrm{CpG})$ or polyriboinosinic:polyribocitidylic acid (polyI:C) need to enter the endosome to stimulate their cognate toll-like receptors (TLR) TLR9 and TLR3, respectively. It is also the endosome where

Key words: microspheres, incomplete Freund's adjuvant, stimulation of T-cell proliferation, in vivo cytotoxicity, cancer

Abbreviations: $\mathrm{CpG}$ : cytosine-phosphorothioate-guanine oligodeoxynucleotides; DC: dendritic cell; IFA: incomplete Freund's adjuvant; MS: microspheres; OVA: ovalbumin; PBS: phosphatebuffered saline; PLGA: poly(D,L-lactide-co-glycolide); polyI:C: polyriboinosinic:polyribocitidylic acid; TLR: toll-like receptors Grant sponsor: Deutsche Krebshilfe; Grant number: 107943 Conflict of interest: None

Correspondence to: Marcus Groettrup, Lehrstuhl Immunologie, Universität Konstanz, Universitätsstrasse 10, D-78457 Konstanz, Germany, Tel.: +49 -7531-882130, Fax: +49-7531-883102, E-mail: marcus.groettrup@uni-konstanz.de antigens have to be delivered for cross presentation on MHC class I molecules and direct presentation on MHC class II molecules. ${ }^{1}$ To achieve an effective T-cell stimulation, it is beneficial if antigen and adjuvant reach the same endosome, ${ }^{2,3}$ which makes their joint uptake highly desirable.

The progress in the development of antigen delivery systems that achieve this aim is lagging behind, thereby hampering a successful introduction of immunotherapy into clinical practice. For numerous clinical trials, antigens and pattern molecules are incorporated into a formulation, which has been introduced over 60 years ago, i.e., incomplete Freund's adjuvant (IFA). ${ }^{4}$ IFA is a water-in-oil emulsion, which can be mixed with antigens and pattern molecules. Although IFA/ antigen emulsions elicit long-lasting IgG responses, they also stimulate the activation of CTL and T-helper cells. ${ }^{5,6}$ In spite of such desired properties, IFA is not approved for routine immunotherapy in humans, but is merely used in investigational clinical trials. Recent studies described severe local skin reactions, sterile abscesses and cysts, inflammations and persistent painful granulomas at the injection site, when using IFA or a formulation similar to IFA (Montanide ISA-51). Moreover, oil-induced neoplasms have been observed. ${ }^{8}$ The need to develop potent and well-defined antigen delivery systems is therefore self-evident.

We and other groups have investigated microspheres (MS) consisting of poly(D,L-lactide-co-glycolide) (PLGA) as antigen delivery system targeting dendritic cells (DCs) and macrophages in vitro and in vivo. ${ }^{9-13}$ PLGA is approved for 
use in humans, e.g., for delivering drugs and as material for biodegradable surgical sutures. ${ }^{14}$ PLGA can be used to microencapsulate peptides, proteins, RNA or DNA by several procedures, such as spray drying, which produces MS of a size of $1-5 \mu \mathrm{m} .{ }^{15}$ Such size range is ideal for the phagocytic uptake by DC in vitro. ${ }^{16}$ PLGA hydrolyses in aqueous environment during several weeks thereby releasing slowly microencapsulated substances. Uptake of PLGA-MS by human monocyte-derived DC in vitro does not negatively affect their survival, migration, cytokine release or T-cell stimulation. ${ }^{16}$ After being ingested by DC, PLGA-MS release microencapsulated antigens intracellularly for processing and presentation on MHC class I and II molecules. ${ }^{17}$ Especially, cross presentation may be at least a 100 -fold more sensitive in vitro if the antigen is taken up in association with PLGA-MS when compared to a soluble form.

On the basis of these in vitro properties, we and others have investigated the potency of PLGA-MS for T-cell stimulation in the mouse model. ${ }^{18-23}$ With a single injection of MS-OVA/CpG, OVA-specific CTL responses mounting up to $8 \%$ of $\mathrm{CD}^{+}$cells could be obtained; the CTL responses mediated lysis of target cells in vivo and in vitro, production of IFN- $\gamma$ and protection of mice from infection with vaccinia virus. ${ }^{18}$ In our study, we tested the antitumor response achievable with PLGA-MS and compared it systematically with an IFA formulation. We found that PLGA-MS-based immunotherapy elicited long-lasting antibody titers, cytolytic and T-helper cell responses and protected mice from growth of melanoma and thymoma in a protective and therapeutic setting. The efficacy was equivalent or even better than that achieved with IFA, which underlines the potential of PLGAMS for immunotherapy of cancer.

\section{Material and Methods Preparation of microspheres}

Microspheres (MS) were prepared from 14-kDa PLGA 50:50 (Resomer RG502H, Boehringer Ingelheim, Ingelheim, Germany). The antigens and TLR ligands were microencapsulated by spray drying as described elsewhere. ${ }^{15}$ Briefly, $50 \mathrm{mg}$ ovalbumin (OVA, Grade V, Sigma) and $5 \mathrm{mg}$ CpG oligodeoxynucleotides with a phosphothioate backbone (CpGODN 1826, Microsynth, Balgach, Switzerland) or $0.5 \mathrm{mg}$ polyI:C (Calbiochem, VWR, Dietikon, Switzerland) (MS polyI:C) were dissolved in $0.5 \mathrm{ml} 0.1 \mathrm{M} \mathrm{NaHCO}_{3}$ (aqueous phase) and mixed with $1 \mathrm{~g}$ of PLGA dissolved in $20 \mathrm{ml}$ of dichloromethane (organic phase). The two phases were emulsified by ultrasonication (Hielscher, UP200 H, Ampl. 40\%) for $10 \mathrm{sec}$ on ice. The obtained w/o dispersion was immediately spray dried (Büchi, Mini Spray-Dryer 191, Büchi, Flawil, Switzerland) at a flow rate of $2 \mathrm{ml} / \mathrm{min}$ and inlet/outlet temperatures of $40^{\circ} \mathrm{C} / 37^{\circ} \mathrm{C}$. The obtained MS were within a size range of $0.5-5 \mu \mathrm{m}$ for all batches; they were washed out of the spray-dryer's cyclone with $0.05 \%$ poloxamer 188 (Synperonic $® F 68$, Serva Electrophoresis, Heidelberg, Germany), collected on a cellulose acetate membrane filter and dried under reduced pressure $(20 \mathrm{mbar})$ for $18 \mathrm{hr}$ at room temperature. The encapsulation efficacy of OVA in MS-OVA/CpG was determined to be $47 \% \pm 2 \%$ according to a previously described protocol. ${ }^{18}$ The release of polyI:C from MS-polyI:C was determined to be $37.8 \% \pm 6.6 \%$. PLGA-MS were stored under desiccation at $4^{\circ} \mathrm{C}$. Immediately before use, MS were dispersed in PBS by ultrasonication for $30 \mathrm{sec}$.

\section{Mice and immunizations}

OT-I mice were obtained from Dr. Ying Waeckerle-Men (University of Zurich, Switzerland). O'T-II/Thyl.1 mice were obtained from Prof. Thomas Brocker (LMU Munich, Germany). C57BL/6 mice $\left(\mathrm{H}-2^{\mathrm{b}}\right)$ were purchased from Charles River Laboratories. All mice were kept in a specific pathogenfree facility and used at 6-10 weeks of age. Animal experiments were approved by Regierungspräsidium Freiburg. For Figures $4-6$, mice were immunized s.c. at the base of the tail [which yielded superior CTL responses compared to i.p. immunization as previously determined (data not shown)] either with a mixture of $5 \mathrm{mg}$ PLGA-MS loaded with OVA $(250 \mu \mathrm{g})$ and CpG-ODN $(25 \mu \mathrm{g})$ and $5 \mathrm{mg}$ MS-polyI:C (2.5 $\mu \mathrm{g})$ or the corresponding amounts of OVA, CpG-ODN and polyI:C in PBS:IFA (1:1) (IFA). This dose was previously shown to yield optimal CTL responses. ${ }^{18}$ For Figures 1-3, mice were only immunized with MS-OVA/CpG as outlined above. Control mice were either treated with empty MS (empty) or left untreated (naïve). All injections were performed at a single site with a total volume of $200 \mu$ l. The recombinant vaccinia virus expressing $\mathrm{OVA}^{24}$ was obtained from Dr. J. Yewdell (NIAID, Bethesda, MD) and propagated as previously described ${ }^{24}$; it was injected i.p. at a dose of $2 \times$ $10^{6}$ p.f.u.

\section{Statistical analyses}

For statistical analyses, groups from similar experiments were pooled and analyzed for significant differences as indicated in the graph. The $p$ values for experiment composites are given in the figure legends.

\section{Tumor cell lines and inoculation of mice}

EL-4 thymoma cells ${ }^{25}$ and the OVA-expressing transfectant EG-7 ${ }^{26}$ were kindly provided by Dr. Wolfram Osen (DKFZ Heidelberg) and kept in RPMI medium. The OVA transfectant of B16F10 melanoma, MO-5, was kindly provided by Dr. Antje Heit ${ }^{19}$ (LMU Munich) and kept in DMEM medium. All media contained GlutaMAX, 10\% fetal calf serum (FCS) and $100 \mathrm{U} / \mathrm{ml}$ of penicillin/streptomycin (P/S). To produce the clone Vf10, TrampC2 cells $^{27}$ were transfected with a plasmid encoding for full-length, cytosolic OVA. Vf10 cells were kept in DMEM (-pyruvate) and complemented with 5\% FCS, $5 \% \mathrm{Nu}$-serum, $5 \mu \mathrm{g} / \mathrm{ml}$ of insulin, $10^{-8} \mathrm{M}$ dihydrotestosterone and $1 \% \mathrm{P} / \mathrm{S}(5 \mathrm{ml})$. To maintain the expression of OVA in EG-7, MO-5 and Vf10 transfectants, the media were supplemented with $\mathrm{G} 418(0.2 \mathrm{mg} / \mathrm{ml})$. For the inoculation of 

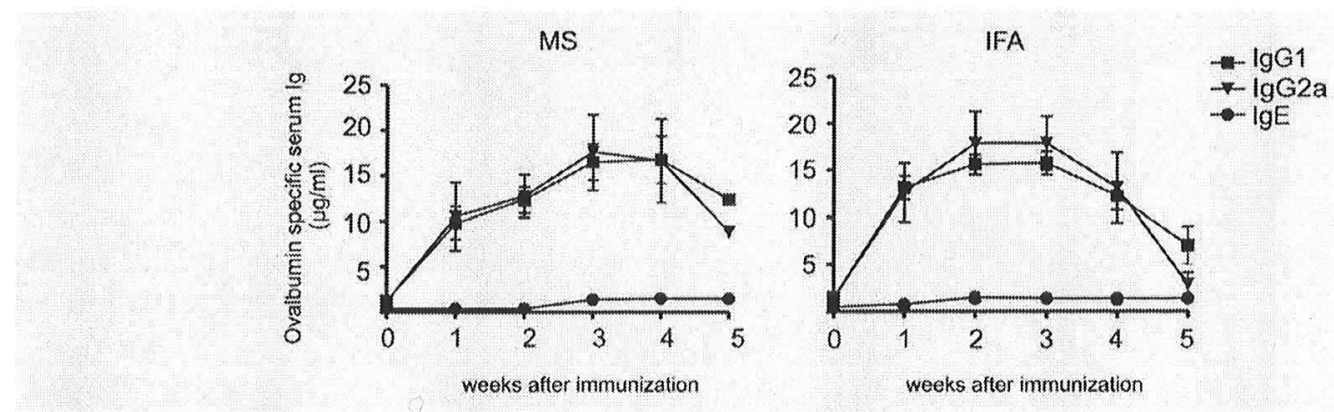

Figure 1. Serum levels of ovalbumin-specific Ig after a single injection of either MS-OVA/CpG or OVA and CpG in IFA. C57BL/6 mice $(n=6)$ were immunized with $5 \mathrm{mg}$ of MS-OVA/CpG containing ovalbumin $(250 \mu \mathrm{g})$ and $\mathrm{CpG}$ oligonucleotide $(25 \mu \mathrm{g})$ or the same amounts of OVA and $\mathrm{CPG}$ in IFA. The sera were analyzed for ovalbumin-specific IgG1 (squares), IgG2a (triangles) or IgE (dots) at indicated time points. The values represent the means \pm SEM of ovalbumin-specific $\mathrm{lg}$ in $\mu \mathrm{g} / \mathrm{ml}$ from two independent experiments.

mice, a number of $5 \times 10^{5}$ EG-7 or MO-5 cells, which we titrated to yield optimal tumor growth, were injected subcutaneously into the right flank. Mice were either treated 6 days earlier (protective setting) or as soon as palpable tumor occurred (therapeutic setting). For lung metastasis in the protective setting, $5 \times 10^{4}$ melanin-expressing MO-5 cells were injected into the tail vein at Day 6 after treatment. After 14 days, mice were sacrificed, lungs were removed and visible metastases were counted. Therapeutic treatment of lung metastases started 1 week after inoculation with $2 \times 10^{4}$ melanin-expressing MO-5 cells via the tail vein, and mice were sacrificed after 2 additional weeks.

\section{Intracellular cytokine staining and MHC tetramer staining}

Splenocytes were isolated and incubated with or without 10 $\mu \mathrm{M}$ SIINFEKL peptide (Eurogentec, Cologne) in the presence of brefeldin A $\left(10 \mu \mathrm{g} / \mathrm{ml}\right.$, Sigma-Aldrich) for $5 \mathrm{hr}$ at $37^{\circ} \mathrm{C}$. After washing, cells were stained with PE-Cy5-conjugated anti-mouse $\mathrm{CD} 8 \alpha$ antibody (BD Biosciences Pharmingen, Clone 53-6.7) for $20 \mathrm{~min}$ at $4^{\circ} \mathrm{C}$. The cells were washed and fixed with $4 \%$ paraformaldehyde in PBS for $10 \mathrm{~min}$ at room temperature. After washing the cells twice with PBS, cells were labeled intracellularly with FITC-conjugated rat antiIFN- $\gamma$ antibody (clone XGM1.2, diluted in PBS/0.1\% saponin) at $4{ }^{\circ} \mathrm{C}$ overnight. The next day, cells were washed and resuspended in PBS for flow cytometry. Background levels of each sample (without peptide) were subtracted. The $\mathrm{H}-2 \mathrm{~K}^{\mathrm{b}}$ / SIINFEKL tetramers used in our study were kindly provided by Prof. Dirk Busch (TU Munich). For MHC tetramer staining, splenocytes were stained with PE-labeled SIINFEKL/H$2 \mathrm{~K}^{\mathrm{b}}$-tetramer for $20 \mathrm{~min}$ at $37^{\circ} \mathrm{C}$ and subsequently with $\mathrm{PE}$ Cy5-conjugated rat anti-CD8 IgG for $20 \mathrm{~min}$ at $4^{\circ} \mathrm{C}$. Cells were washed twice and measured by flow cytometry.

\section{In vivo cytotoxicity assay}

CTL activity in vivo was assessed exactly as described elsewhere. ${ }^{28}$ The percentage of specific cytolysis was calculated as follows: $100-[(\%$ peptide pulsed cells in vaccinated mice $/ \%$ unpulsed cells in vaccinated mice)/(\% peptide pulsed cells in control mice $/ \%$ unpulsed cells in control mice) $] \times 100$.

\section{Ex vivo cytotoxicity assays}

For the classical chromium release assay (Fig. 5a), C57BL/6 mice were immunized either by a mixture of PLGA-MS or the corresponding components in IFA. Six days later, splenocytes were used as effectors in a primary chromium release assay as previously described. ${ }^{29}$ EL-4 $\left(\mathrm{H}-2^{\mathrm{b}}\right)$ (negative control) or EG-7 cells served as targets. For measurement of ex vivo cytolytic activity of splenocytes from vaccinated C57BL/ 6 mice in Figure $5 b$, a time-resolved fluorometric assay based on the DELFIA ${ }^{\circledR}$ EuTDA reagent (PerkinElmer) was used. ${ }^{30}$ Briefly, $5 \times 10^{4}$ Vf10 cells served as target cells and were labeled with BaTDA (PerkinElmer). After $4 \mathrm{hr}$ of coincubation, supernatants were mixed with europium solution for $1 \mathrm{hr}$ and measured at ex/em wavelengths of $340 / 615 \mathrm{~nm}$ and a lag time of 200 nsec. Specific cytolysis was calculated for both assays as follows: [(experimental release - spontaneous release $) /($ total release - spontaneous release $)] \times 100 \%$.

\section{ELISA}

For detection of OVA-specific immunoglobulin (Ig) in plasma, blood samples were collected at indicated time points after immunization using a heparin-coated microvette (Sarstedt, Nümbrecht, Germany) and spun down for $5 \mathrm{~min}$ at 1,000g. Ninety-six-well ELISA plates were coated with 0.1 $\mathrm{mg} / \mathrm{ml}$ OVA in PBS overnight at $4^{\circ} \mathrm{C}$. The next day, plates were washed three times with PBS-Tween $20(0.05 \%)$, and serum samples were applied for $2 \mathrm{hr}$ at room temperature. For detection of mouse Ig isotypes, the mouse-Ig-isotyping-kit (BD Bioscience) was used. For detection of IL- 4 and IFN- $\gamma$, the respective kit (mouse IFN- $\gamma /$ IL- 4 ELISA set, BD Bioscience) was used, following the manufacturer's instruction.

\section{Proliferation assay}

Proliferation of lymphocytes was measured via CFSE dilution. Immunized mice received $1 \times 10^{7}$ CFSE $(10 \mu \mathrm{M})$-labeled splenocytes derived from OT-I or OT-II/Thyl.1 mice. 

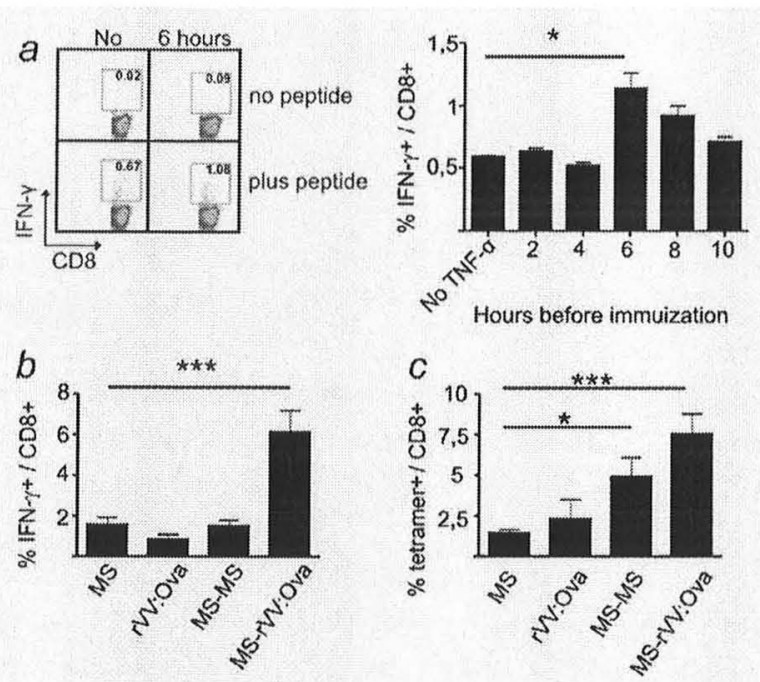

Figure 2. Effects of pretreatment of the injection site and primeboost vaccination on CTL responses. (a) C57BL/ 6 mice $(n=3)$ were pretreated with $200 \mathrm{ng}$ TNF- $\alpha$ at the site of injection at 2, 4, 6,8 or $10 \mathrm{hr}$ before immunization with $5 \mathrm{mg}$ of MS-OVA/CpG containing ovalbumin ( $50 \mu \mathrm{g} / \mathrm{mg} \mathrm{MS}$ ) and CpG-ODN ( $5 \mu \mathrm{g} / \mathrm{mg} \mathrm{MS})$. Control animals were left untreated (No). Six days after immunization, splenocytes were analyzed by IFN- $\gamma$-ICS. Two representative flow cytometry dot plots (left panels) and mean values (right panel) from one out of three experiments with similar outcomes are shown. Values (mean \pm SEM) are given in percent IFN- $\gamma^{+}$cells of $\mathrm{CD}^{+}$cells. Background signals (no peptide) were subtracted. The $p$ value was calculated by a Student's $t$-test $(p=$ 0.0317). ( $b, c)$ Effects of booster immunizations: C57BL/6 mice ( $n$ $=2$ ) received single injections of $M S-O V A / C p G(M S)$ or recombinant vaccinia virus expressing ovalbumin (rV:OVA) or additional booster immunizations after 4 weeks (MS-MS or MSrVV:OVA). Six days after the last immunization, splenocytes were analyzed by ICS $(b)$ or SIINFEKL/H-2 $\mathrm{K}^{b}$-tetramer staining (c). Results are from one out of three experiments with similar outcomes. Values (mean $\pm \mathrm{SEM}$ ) for $(b)$ are given in percent IFN $-\gamma^{+}$of $\mathrm{CD} 8^{+}$ lymphocytes. Background signals (no peptide) were subtracted. The $p$ value was calculated by a Student's $t$-test $(p=0.0001)$. Values (mean \pm SEM) in $(c)$ are given in percent SIINFEKL/H-2 $\mathrm{K}^{\mathrm{b}}$. tetramer ${ }^{+}$cells of $\mathrm{CD}^{+}$lymphocytes. The background (naïve mouse) was subtracted. The $p$ values were calculated by a Student's $t$-test $\left({ }^{\star} p=0.0153 ;{ }^{* \star} p=0.0002\right)$.

For transfer of $\mathrm{CD}^{+}$lymphocytes, OT-I splenocytes were treated with erythrocyte-lysis buffer (155 $\mathrm{mM} \mathrm{NH}_{4} \mathrm{CL}$ and $0.01 \mathrm{M}$ Tris) for $5 \mathrm{~min}$ at room temperature. $\mathrm{CD}^{+}$cells were magnetically sorted, labeled with CFSE and injected i.v. into Thyl.1 recipient mice. Three days later, spleens of Thy1.1 recipient mice were stained for Thy $1.2^{+}$cells [PECy5-conjugated anti-mouse CD90.2 (Thy1.2), eBioscience, Clone 53-2.1] for $20 \mathrm{~min}$ at $4^{\circ} \mathrm{C}$, washed with PBS and analyzed by flow cytometry. For transfer of OT-II/Thy1.1 spleno-



Figure 3. Production of IFN- $\gamma$ and IL- 4 by $\mathrm{CD}^{+}$cells after immunization with MS-OVA/CpG or OVA and CpG in IFA. C57BL/6 mice $(n=10)$ were immunized with $5 \mathrm{mg} \mathrm{MS}$ containing $250 \mu \mathrm{g}$ ovalbumin and $25 \mu \mathrm{g}$ CpG oligonucleotides (black bars) or the corresponding amounts of ovalbumin and CpG-ODN in IFA (gray bars). Control groups were immunized with corresponding amounts of encapsulated $\mathrm{CpG}$ alone or $\mathrm{CpG}$ alone in IFA. After 6 days, splenocytes were isolated and magnetically sorted for $\mathrm{CD}_{4}^{+}$cells. $5 \times 10^{5} \mathrm{CD}^{+}$cells were restimulated by plate-bound anti-CD3 and anti-CD28 Ig for $18 \mathrm{hr}$. Supernatants were analyzed for IL-4 and IFN- $\gamma$ by ELISA. Background levels of naive mice were subtracted. Values are given in $\mathrm{ng} / \mathrm{ml}$ (mean $\pm \mathrm{SEM}$ ). Results are a summary of two independent experiments with similar outcomes.

cytes, spleens of OT-II/Thy1.1 mice were homogenized and treated with erythrocyte-lysis buffer for $5 \mathrm{~min}$ at room temperature. Cells were labeled with CFSE and injected i.v. into C57BL/6 recipient mice. Three days later, spleens of recipient mice were harvested, homogenized and stained for Thy1.1 [PE-Cy5-conjugated anti-mouse CD90.1 (Thyl.1) Ig, eBioscience, Clone HIS51] and for CD4 (PE-conjugated antimouse CD4 Ig, eBioscience, Clone RMA4-5) for $20 \mathrm{~min}$ at $4^{\circ} \mathrm{C}$. CD4/Thy1.1 double positive cells were analyzed for CFSE fluorescence.

\section{Results}

Humoral response to single injection of PLGA-MS

To determine the humoral immune response to PLGA-MS, mice received a single injection of MS containing OVA and CpG oligonucleotides (MS-OVA-CpG) or the same amount of antigen and TLR ligand in IFA. The OVA-specific Ig isotype concentrations indicate a robust IgG1 and IgG2a response but only low levels of IgE (Fig. 1). Empty MS showed no significant increase of Ig isotypes within 4 weeks (data not shown). The maximum concentration of about $18 \mu \mathrm{g} / \mathrm{ml}$ of OVA-specific IgG1 and IgG2a was reached after 3 weeks and stayed at that level until the end of the experiment after 5 weeks post- 
MS-based vaccination, whereas we observed a marked decline of the titers after the 5th week when IFA-based immunization was performed. The relative levels of OVA-specific IgG1 to IgG2a suggested a balanced $\mathrm{Th}_{1} / \mathrm{Th}_{2}$ response.

\section{Enhancement of CTL responses by TNF- $\alpha$ pretreatment and prime-boost vaccination}

We tested two options to enhance the CTL response to PLGA-MS: ( $i$ ) by pretreatment of the injection site with TNF- $\alpha^{31}$ and (ii) by administering a booster injection. The pretreatment of the injection site with $200 \mathrm{ng}$ TNF- $\alpha$ at least $6 \mathrm{hr}$ before immunization increased the CTL response markedly, and this effect was still visible when the pretreatment was performed up to $8 \mathrm{hr}$ before immunization (Fig. 2a). Homologous priming-boosting with $\mathrm{MS}-\mathrm{OVA} / \mathrm{CpG}$ within an interval of 4 weeks failed to increase OVA-specific CTL responses, as determined by intracellular IFN $-\gamma$ staining (ICS), whereas a heterologous boosting using recombinant vaccinia virus expressing OVA improved the CTL response (Fig. 2b). Flow cytometric analysis with SIINFEKL/H-2K $\mathrm{K}^{\mathrm{b}}$ tetramers confirmed the enhanced response after heterologous as opposed to homologous boosting with MS-OVA/CpG, but this read-out evidenced also an effect of a homologous boosting (MS-MS) (Fig. 2c). It was noteworthy that not all tetramer ${ }^{+} \mathrm{CD}^{+}$cells obtained after homologous boosting (MSMS) also showed IFN- $\gamma$ production in the ICS.

\section{IFN- $\gamma$ but no IL-4 production by $\mathrm{CD}^{+}{ }^{+}$splenocytes from PLGA-MS-vaccinated mice}

The propensity for $\mathrm{Th}_{1}$ as opposed to $\mathrm{Th}_{2}$ differentiation after MS-based immunization was further characterized by the IL- 4 and IFN- $\gamma$ concentrations in the supernatant of splenocytes harvested from mice, either immunized with MS-OVA/ $\mathrm{CpG}$ or a formulation of the same substances in IFA. As a control, we used MS or IFA containing CpG oligonucleotides alone. Subsequent to stimulation with anti-CD3 and antiCD28 antibodies in vitro, the supernatants of both splenocyte preparations contained predominantly IFN- $\gamma$ and barely detectable levels of IL-4, arguing for a $\mathrm{Th}_{1}$ bias conferred by both vaccination modes (Fig. 3).

\section{Persistence of CTL and T-helper cell stimulation after PLGA-MS- and IFA-based vaccination}

As PLGA-MS and IFA are expected to create a depot of antigen at the site of injection, we investigated the potential of PLGA-MS- and IFA-based vaccination to induce in vivo proliferation of OVA-specific $\mathrm{CD}^{+}$and $\mathrm{CD}^{+}{ }^{+} \mathrm{T}$ cells derived from OT-1 and OT- 2 mice at several time points after vaccination. To this aim, we vaccinated the mice with either a mixture of MS-OVA/CpG and MS-polyI:C, which we determined to be the most potent vaccination scheme (M. Mueller, unpublished data), or the same amounts of OVA, CpG oligos and polyI:C in IFA. Following CFSE dilution assays, both PLGA-MS- and IFA-based vaccination induced the proliferation of transferred $\mathrm{CD} 4^{+*}$ and $\mathrm{CD} 8^{+} \mathrm{T}$ cells in vivo even

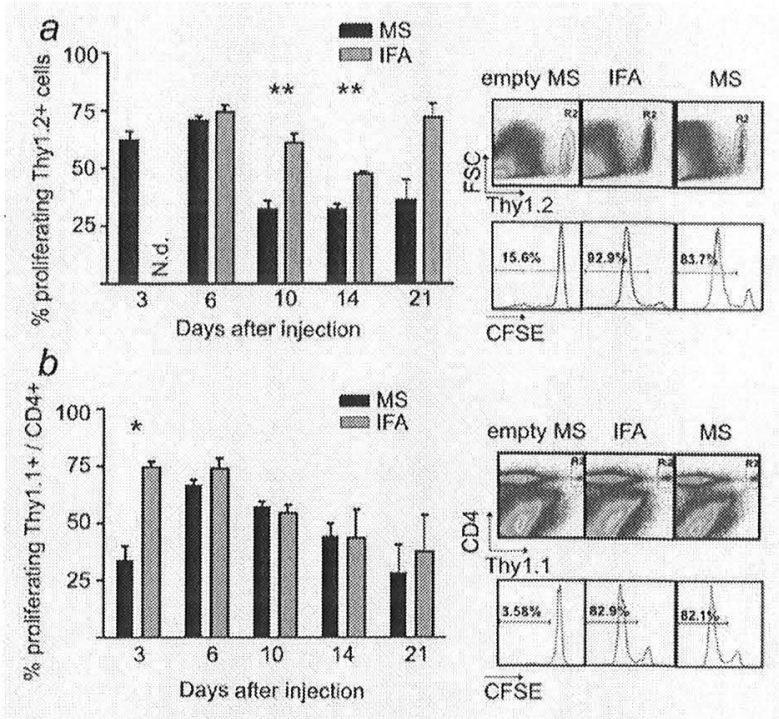

Figure 4. Time course of antigen presentation after immunization with MS and IFA in vivo. (a) Thy1.1 mice $(n=3)$ were immunized with a mixture of MS-OVA/CpG and MS-polyl:C (black bars) or the corresponding amounts of ovalbumin $(250 \mu \mathrm{g}), \mathrm{CpG}-$ ODN $(25 \mu \mathrm{g})$ and polyl:C $(2.5 \mu \mathrm{g})$ in IFA (gray bars). Three days before mice were sacrificed, $1 \times 10^{7}$ sorted $\mathrm{CD}^{+}$and CFSE-labeled splenocytes from OT-I/Thy1.2 mice were injected i.v. into Thy1.1 mice. Then, Thy $1.2^{+}$splenocytes were analyzed for CFSE fluorescence. Representative results from one out of two experiments with similar outcomes are shown (left panel). Values (mean \pm SEM) are given in percent proliferating Thy $1.2^{+}$cells. Background values (PBS-treated mice) were subtracted. The $p$ values were calculated by a Student's $t$-test (Day 10: $p=0.008$; Day 14: 0.009). The right panels show representative flow cytometry dot plots for gating of Thy $1.2^{+}$cells and histograms for CFSE staining of gated cells from three mice. Panels in $(b)$ show evidence for the potential to stimulate proliferation of $\mathrm{CD}_{4}^{+}$cells. C57BL/6 mice $(n=2)$ were immunized as outlined above. Three days before mice were sacrificed, $2 \times 10^{6}$ CFSE-labeled splenocytes from OT-II/Thy1.1 mice were injected i.v. Then, Thy $1.1^{\circ} \mathrm{CD} 4$ splenocytes were analyzed for CFSE fluorescence. Representative results from one out of two experiments with similar outcomes are shown (left panel). Values (mean \pm SEM) are given in percent proliferating Thy $1.1^{+} \mathrm{CD} 4^{+}$cells. The $p$ value was calculated by a Student's $t$-test $(p=0.019)$. The gating of Thy $1.1^{+} \mathrm{CD}^{+}$cells in dot plots and representative histograms for CFSE staining of gated cells is shown on the right.

when the T-cell transfer occurred up to 21 days after inoculation (Figs. $4 a$ and $4 b$ ).

\section{In vivo and ex vivo cytotoxicity elicited by PLGA-MS- and} IFA-based vaccination

The cytotoxic effect of the generated CTLs was assessed by in vivo and ex vivo assays. At 6 days after immunization with 

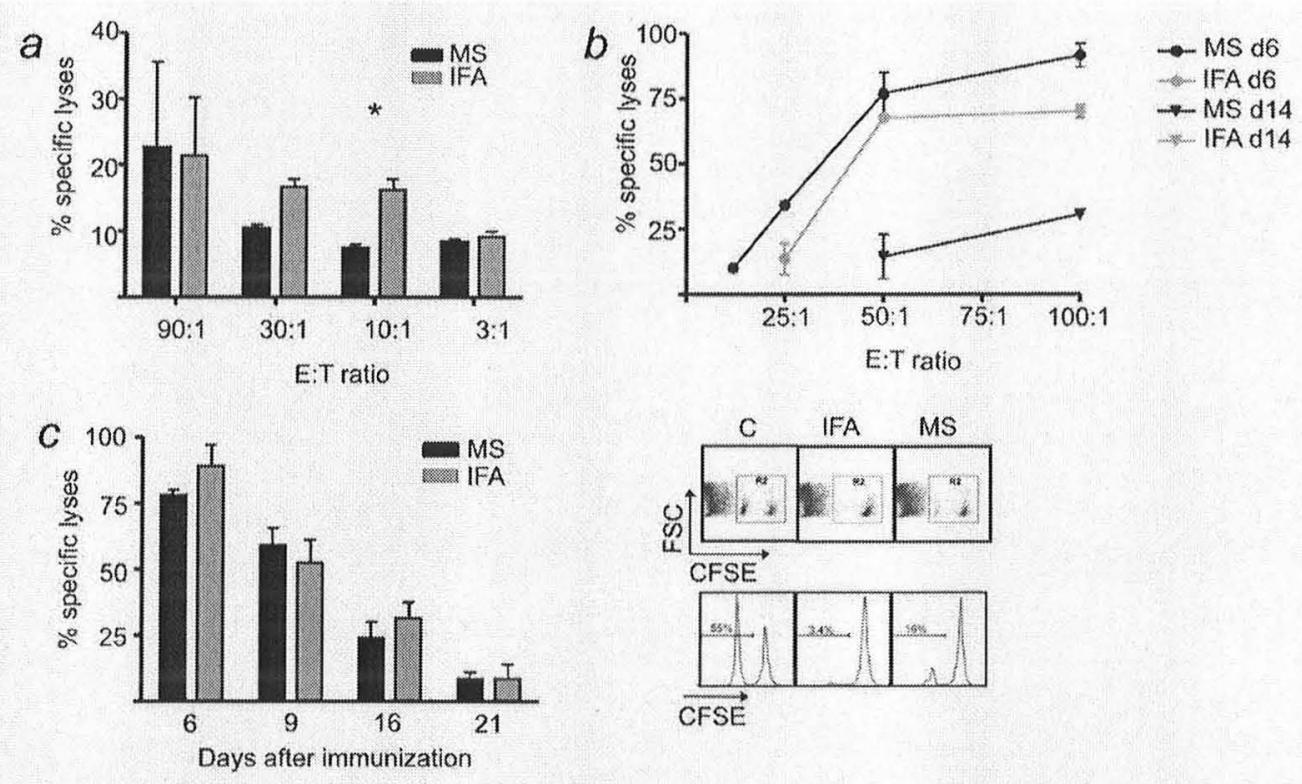

Figure 5. CTL-mediated cytotoxicity elicited by vaccination with PLGA-MS and IFA. (a) Cytotoxicity as determined by 51-chromium release assay. C57BL/6 mice $(n=2)$ were immunized with a mixture of MS-OVA/CpG and MS-polyl:C (black bars) or the corresponding amounts of ovalbumin $(250 \mu \mathrm{g}), \mathrm{CpG}-\mathrm{ODN}(25 \mu \mathrm{g})$ and polyl:C $(2.5 \mu \mathrm{g})$ in IFA (gray bars). On Day 6 , splenocytes were coincubated for $4 \mathrm{hr}$ with 51 chromium-labeled EL-4 or Eg-7 cells expressing ovalbumin. Values (mean \pm SEM) are given in percent-specific lysis. Data from one out of two experiments with similar outcomes are shown. The $p$ value for the E:T ratio of 10:1 was calculated by a Student's $t$-test $(p=0.039)$. (b) Cytotoxicity as determined by BaTDA-cytotoxicity assay described in the Material and Methods section. C57BL/6 mice $(n=2)$ were immunized with a MS mixture (black lines) or IFA (gray lines), as outlined above. Spleens were removed after 6 days (circles) or 14 days (triangles) and coincubated with BaTDA-labeled TrampC2 cells that express ovalbumin (Vf10 cells). Values (mean \pm SEM) are given in percent-specific lysis with indicated effector-to-target ratios. Data from one out of three experiments with similar outcomes are shown. No lysis was found 14 days after vaccination with IFA. (c) In vivo cytotoxicity assay. C57BL/6 mice $(n=3)$ were immunized as described under (a). In vivo cytotoxicity was analyzed on indicated days, and representative dot plots and histograms of gated $\mathrm{CFSE}^{+}$cells are shown (right). Representative data (mean \pm SEM) from one out of three experiments with similar outcomes are shown (left).

either a mixture of MS-OVA/CpG and MS-polyI:C or the same amounts of OVA, CpG oligos and polyI:C in IFA, the IFA formulation elicited a slightly superior ex vivo cytotoxicity in the 51-chromium cytotoxicity assay at effector to target ratios of 30:1 and 10:1 than the PLGA-MS formulation (Fig. 5a). However, in the nonradioactive EuBATDA cytotoxicity assay, the PLGA-MS- and IFA-based vaccinations elicited similar cytolytic responses, yielding a detectable cytolytic response ex vivo even 14 days after immunization (Fig. $5 b$ ). The in vivo cytotoxicity assay showed robust responses at 6 days after PLGA-MS- and IFA-based vaccinations, which remained detectable when target cells were injected 9,16 or even 21 days after vaccination, thus indicating that both vaccine formulations yielded long-lasting cytotoxic responses (Fig. 5c).

\section{Tumor eradication by PLGA-MS- and IFA-based immunotherapy}

For comparing the potential of PLGA-MS- and IFA-based vaccination for tumor immunotherapy, we investigated the antitumor responses to EL-4 thymoma (Fig. 6a) as well as to the very aggressive and fast growing B16F10 melanoma (Fig. $6 b$ ). The corresponding OVA transfectants EG-7 and MO-5 were used in either a protective setting (Fig. 6, left-hand side panels), where therapy was started 6 days before challenge, or in a therapeutic setting (Fig. 6, right-hand side panels), where therapy was started not before palpable tumors had been established. Eighty percent of mice receiving treatment with a mixture of MS-OVA/CpG and MS-polyI:C in a protective setting survived tumor free using EG-7 model, and all MS-treated mice survived in the protective MO-5 model. For comparison, all mice being treated with IFA containing the same ingredients remained tumor free in the EG-7 model, but only $60 \%$ survived inoculation with MO-5 tumor cells. The therapeutic settings showed that both delivery systems had the potency to cure all mice from EG-7 tumors. For MO-5, however, only $20 \%$ of the PLGA-MS group and $30 \%$ of the IFA group survived tumor free. In a further assay, metastases in the lung were established by i.v. injection of melanin-producing MO-5 cells. Here, both IFA and PLGA-MS formulations markedly reduced the number of metastases in the protective setting (Fig. $6 c$ ). The therapeutic setting 
a protective

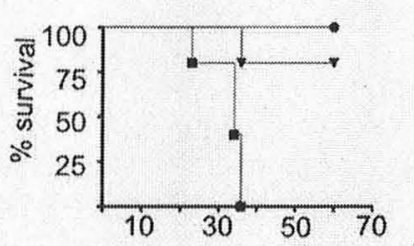

days after injection of EG-7 cells

$b$

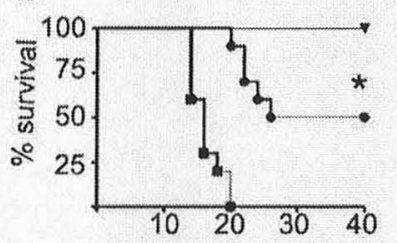

days after injection of $\mathrm{MO}-5$ cells

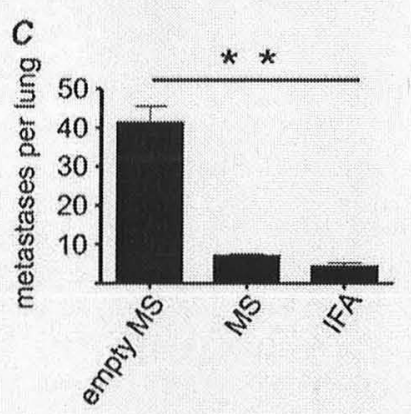

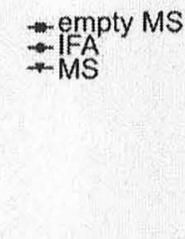



days after injection of MO-5 cells



Figure 6. Antitumor responses elicited with PLGA-MS and IFA against EG-7 thymoma and MO-5 melanoma. $(a, b)$ Left panels show the results from a protective setting. C57BL/6 mice $(n=5)$ were immunized with a mixture of MS-OVA/CpG and MS-polyl:C (triangles) or IFA containing ovalbumin, $\mathrm{CpG}$ oligo and polyl:C in equivalent amounts (dots) or empty PLGA-MS (squares). After 6 days, mice were challenged s.c. with $5 \times 10^{5} \mathrm{EG}-7$ or MO-5 cells. Tumor sizes were measured daily until they reached a mean size of $15 \mathrm{~mm}$. $(a, b)$ Right panels show the results from a therapeutic setting. C57BL/6 mice $(n=10)$ were challenged s.c. with $5 \times 10^{5} \mathrm{EG}-7$ or MO- 5 cells. When palpable tumors were established, mice were immunized with a mixture of MS (triangles), IFA (dots) or empty PLGA-MS (squares), as outlined above. The $p$ value was calculated by a survival curve analysis $(p=0.0496)$. (c) Left panel: metastatic tumor challenge in the protective setting. C57BL/6 mice $(n=3)$ were immunized as outlined above. After 6 days, mice were challenged i.v. with $5 \times 10^{4}$ melanin-expressing MO-5 cells. After 2 weeks, mice were sacrificed and lungs removed. Visible metastases were counted and are given as means \pm SEM. (c) Right panel: metastatic tumor challenge in the therapeutic setting. C57BL/6 mice $(n=3)$ were challenged i.v. with $2 \times 10^{4}$ MO-5 cells. After 1 week, mice were immunized as described above. After 14 days, mice were sacrificed and lungs removed. Metastases numbers are given as means \pm SEM. All graphs show one representative experiment out of three with similar outcomes. The $p$ values were calculated by a oneway analysis of variance for the protective setting $(p=0.0017)$ and for the therapeutic setting $(p=0.03)$.

showed a clear benefit for the group being treated with PLGA-MS when compared to the IFA group. Taken together, we could show that vaccination with PLGA-MS stimulates a robust immune response that has the potency to eradicate solid tumors and to prevent metastasis formation at an extent equivalent or superior to that of IFA.

\section{Discussion}

The pivotal function of DCs for T-cell priming has spurred tremendous efforts to target tumor antigens for uptake and presentation by DC. In the last decade, great efforts have been invested to cultivate autologous human DC in vitro to pulse them with antigen and inject them into patients. ${ }^{32-36}$ This approach, besides yielding largely disappointing clinical results, is very labor and cost intensive. An interesting alternative is the in vivo delivery of antigens and immunostimulants to DC for which IFA formulations have been used most frequently.

Admittedly, IFA formulations have been improved in terms of safety and tolerance by the patients. MF-59, e.g., is 
an emulsified squalene in combination with Tween and Span85, a fatty acid ester of polyhydric alcohol, which stabilizes the water in oil emulsion. Besides alum, MF-59 is the only carrier system approved for clinical use in humans. ${ }^{37,38}$ Nevertheless, both alum and MF-59 are known to favor Th2 responses and show poor efficacy in regard to antigen or adjuvant depot formation. ${ }^{39,40}$ In combination with Th1-polarizing adjuvants like monophosphoryl lipid A (MPL), ${ }^{41}$ a detoxified LPS derivate binding TLR4 ${ }^{42}$ or QS-21, ${ }^{43}$ a subfraction of Quil-A, both have been successfully used in malaria vaccines, in HPV as well as in HIV studies. ${ }^{40,44-46}$

In our study, we investigated the potency of PLGA-MS based vaccination in immunotherapy of mice against two model tumors. We showed that PLGA-MS were equal or superior to IFA-based vaccination currently used in clinical trials. ${ }^{5,47,48}$ A major rationale for developing PLGA-MS as antigen delivery systems resided on their capacity to elicit strong antibody responses after a single administration without the need of booster immunizations. ${ }^{49}$ We could also show that high titers of OVA-specific IgG1 and IgG2a are obtained by a single vaccination and that these titers are maintained for several weeks (Fig. 1). Systemic IgE levels remained low arguing against an enhanced risk of allergic reactions. We hypothesized that the slow biodegradation of PLGA-MS over several weeks would allow the maintenance of an antigen depot, which might sustain CTL responses over an extended period of time. Indeed, the T-cell transfer experiments demonstrated that both IFA- and PLGA-MS-based vaccinations sustain antigen presentation for over 21 days in vivo (Fig. 4). More importantly, the induced CTLs were capable to lyse antigen-charged cells for up to 21 days after immunization. This result came as a surprise, because we had previously shown by IFN- $\gamma$ ICS that PLGA-MS-based vaccination led to a peak of IFN- $\gamma$-producing $\mathrm{CD}^{+}$cells on Day 6 after vaccination and declined rapidly thereafter. ${ }^{18}$ Hence, the capacity to lyse target cells is sustained longer than that of producing IFN- $\gamma$. We were concerned that the persistence of antigen in PLGA-MS would induce regulatory $\mathrm{T}$ cells, but no accumulation of $\mathrm{CD}^{+}{ }^{+} \mathrm{CD} 25^{+} \mathrm{FOXP}^{+}{ }^{+} \mathrm{T}$ cells was observed over time following PLGA-MS-based vaccination (M. Mueller, unpublished data). Consistently, IFN- $\gamma$ production and the switch to IgG2a was much more prominent after IFA- or PLGA-MS-based vaccination than IL-4 production or the switch to IgE. It therefore appears that both formulations induce a $\mathrm{Th}_{1}$ differentiation, which is probably due to $\mathrm{CpG}$ oligo and polyI:C coadministered with the antigen.

In our efforts to optimize PLGA-MS-based vaccination, two parameters turned out to be helpful for enhancing the $\mathrm{Th}_{1}$ responses to PLGA-based vaccination: $(i)$ the preconditioning of the injection site with TNF- $\alpha^{31}$ and (ii) the use of two different pattern molecules, CpG and polyI:C. Also, the capacity to maintain IFN- $\gamma$ production by antigen-specific $\mathrm{CD}^{+}$cells by a heterologous boost with recombinant vaccinia virus is an important finding of our study, which could be exploited in clinical trials. Both the ability of CTLs to directly lyse tumor cells as well as the production of IFN- $\gamma$ by CTL and Th1 cells will most likely contribute to the observed antitumor responses (Fig. 6).

Finally, it is interesting to compare the efficacy of the antitumor response of PLGA-MS- and IFA-based vaccination. Although both vaccination regimes mediated the complete eradication of well-palpable EG-7 thymomas (Fig. 6a), the protection against this tumor and the aggressive MO-5 melanoma was enhanced when PLGA-MS-based vaccination preceded tumor inoculation. Also, the suppression of metastasis formation in the lung after MO-5 inoculation was clearly stronger when PLGA-MS based vaccination was used in a therapeutic setting (Fig. 6c). Our tumor protection and immunotherapy data are consistent with other tumor vaccination studies using PLGA nanoparticles or microparticles containing protein antigens and TLR ligands, which showed that the coencapsulation of antigen and pattern molecules markedly improves the antitumor response ${ }^{21}$ and even allowed the eradication of preexisting B16F10 melanoma. ${ }^{19}$ Taken together, PLGA-MS-based vaccination combines excellent biocompatibility with high and long-lasting antitumor responses resulting in powerful tumor eradication in mice. Therefore, the investigation of PLGA-MS-based vaccination in a phase 1 clinical trial of immunotherapy against cancer is clearly warranted.

\section{Acknowledgements}

The authors thank Dirk Busch for the contribution of MHC tetramers, Wolfram Osen and Antje Heit for cell lines, Jonathan Yewdell for recombinant vaccinia virus and Thomas Brocker and Ying Waeckerle-Men for transgenic mice. This study was supported by a grant from Deutsche Krebshilfe to M.G.

\section{References}

1. Carbone FR, Bevan MJ. Class I-restricted processing and presentation of exogenous cell-associated antigen in vivo. $J$ Exp Med 1990;171:377-87.

2. Blander JM, Medzhitov R. Toll-dependent selection of microbial antigens for presentation by dendritic cells. Nature 2006;440:808-12.

3. Yarovinsky F, Kanzler H, Hieny S, Coffman RL, Sher A. Toll-like receptor recognition regulates immunodominance in an antimicrobial CD4+ T cell response. Immunity 2006;25:655-64.

4. Freund J. The effect of paraffin oil and mycobacteria on antibody formation and sensitization: a review. Am J Clin Pathol 1951;21:645-56.

5. Speiser DE, Lienard D, Rufer N, RubioGodoy V, Rimoldi D, Lejeune F, Krieg AM, Cerottini JC, Romero P. Rapid and strong human $\mathrm{CD}^{+} \mathrm{T}$ cell responses to vaccination with peptide, IFA, and $\mathrm{CpG}$ oligonucleotide 7909. J Clin Invest 2005; 115:739-46.

6. Moss RB, Diveley J, Jensen F, Carlo DJ. In vitro immune function after vaccination with an inactivated, gp120-depleted HIV-1 antigen with immunostimulatory oligodeoxynucleotides. Vaccine 2000;18: 1081-7.

7. Toledo H, Baly A, Castro O, Resik S, Laferte J, Rolo F, Navea L, Lobaina L, Cruz O, Miguez J, Serrano T, Sierra B, et al. A 
phase I clinical trial of a multi-epitope polypeptide TAB9 combined with Montanide ISA 720 adjuvant in non-HIV1 infected human volunteers. Vaccine 2001; 19:4328-36.

8. Gupta RK, Relyveld EH, Lindblad EB, Bizzini B, Ben-Efraim S, Gupta CK. Adjuvants-a balance between toxicity and adjuvanticity. Vaccine 1993;11:293-306.

9. WaeckerleMen Y, Groettrup M. PLGA microspheres for improved antigen delivery to dendritic cells as cellular vaccines. $A d v$ Drug Deliv Rev 2005;57:475-82.

10. Sharp FA, Ruane D, Claass B, Creagh E, Harris J, Malyala P, Singh M, O'Hagan DT, Petrilli V, Tschopp J, O'Neill LA, Lavelle EC. Uptake of particulate vaccine adjuvants by dendritic cells activates the NALP3 inflammasome. Proc Natl Acad Sci USA 2009;106:870-5.

11. Malyala P, O'Hagan DT, Singh M. Enhancing the therapeutic efficacy of $\mathrm{CpG}$ oligonucleotides using biodegradable microparticles. Adv Drug Deliv Rev 2009; 61:218-25.

12. Akagi T, Wang $\mathrm{X}$, Uto T, Baba $\mathrm{M}$, Akashi $M$. Protein direct delivery to dendritic cells using nanoparticles based on amphiphilic poly(amino acid) derivatives. Biomaterials 2007;28:3427-36.

13. Guillon C, Mayol K, Terrat C, Compagnon C, Primard C, Charles MH, Delair T, Munier S, Verrier B. Formulation of HIV-1 Tat and p24 antigens by PLA nanoparticles or MF59 impacts the breadth, but not the magnitude, of serum and faecal antibody responses in rabbits. Vaccine 2007;25: 7491-501.

14. Johansen P, Men Y, Merkle HP, Gander B. Revisiting PLA/PLGA microspheres: an analysis of their potential in parenteral vaccination. Eur J Pharm Biopharm 2000; 50:129-46.

15. Waeckerle-Men Y, Gander B, Groettrup M. Delivery of tumor antigens to dendritic cells using biodegradable microspheres. Methods Mol Med 2005;109:35-46.

16. Waeckerle-Men Y, Scandella E, Allmen EU, Ludewig B, Gillessen S, Merkle HP, Gander B, Groettrup M. Phenotype and functional analysis of human monocytederived dendritic cells loaded with biodegradable poly(lactide-co-glycolide) microspheres for immunotherapy. $J$ Immunol Method 2004;287:109-24.

17. Waeckerle-Men $\mathrm{Y}$, UetzvonAllmen E, Gander B, Scandella E, Schlosser E Schmidtke G, Merkle HR, Groettrup M. Encapsulation of proteins and peptides into biodegradable poly(D,L-lactide-co-glycolide) microspheres prolongs and enhances antigen presentation by human dendritic cells. Vaccine 2006;24:1847-57.

18. Schlosser E, Mueller M, Fischer S, Basta S, Busch DH, Gander B, Groettrup M. TLR ligands and antigen need to be coencapsulated into the same biodegradable microsphere for the generation of potent cytotoxic $\mathrm{T}$ lymphocyte responses. Vaccine 2008;26: 1626-37.

19. Heit A, Schmitz F, Haas T, Busch DH, Wagner $\mathrm{H}$. Antigen co-encapsulated with adjuvans efficiently drive protective $\mathrm{T}$ cell immunity. Eur J Immunol 2007;37: 2063-74.

20. Hamdy S, Elamanchili P, Alshamsan A, Molavi O, Satou T, Samuel J. Enhanced antigen-specific primary $\mathrm{CD} 4$ and $\mathrm{CD} 8^{+}$ responses by codelivery of ovalbumin and toll-like receptor ligand monophosphoryl lipid A in poly(D,L-lactic-co-glycolic acid) nanoparticles. J Biomed Mater Res A 2007; 81:652-62.

21. Hamdy S, Molavi O, Ma Z, Haddadi A, Alshamsan A, Gobti Z, Elhasi S, Samuel J, Lavasanifar A. Co-delivery of cancerassociated antigen and Toll-like receptor 4 ligand in PLGA nanoparticles induces potent $\mathrm{CD}^{+} \mathrm{T}$ cell-mediated anti-tumor immunity. Vaccine 2008;26:5046-57.

22. Goforth R, Salem AK, Zhu X, Miles S, Zhang XQ, Lee JH, Sandler AD. Immune stimulatory antigen loaded particles combined with depletion of regulatory Tcells induce potent tumor specific immunity in a mouse model of melanoma. Cancer Immunol Immunother 2009;58: 517-30.

23. San Roman B, Irache JM, Gomez S, Tsapis N, Gamazo C, Espuelas MS. Coencapsulation of an antigen and $\mathrm{CpG}$ oligonucleotides into PLGA microparticles by TROMS technology. Eur J Pharm Biopharm 2008;70:98-108.

24. Restifo NP, Bacik I, Irvine KR, Yewdell JW, McCabe BJ, Anderson RW, Eisenlohr LC, Rosenberg SA, Bennink JR. Antigen processing in vivo and the elicitation of primary CTL responses. J Immunol 1995; 154:4414-22.

25. Gorer PA, Kaliss N. The effect of isoantibodies in vivo on three different transplantable neoplasms in mice. Cancer Res 1959;19:824-30.

26. Moore MW, Carbone FR, Bevan MJ. Introduction of soluble protein into the class I pathway on antigen processing and presentation. Cell 1988;54:777-85.

27. Foster BA, Gingrich JR, Kwon ED, Madias C, Greenberg NM. Characterization of prostatic epithelial cell lines derived from transgenic adenocarcinoma of the mouse prostate (TRAMP) model. Cancer Res 1997;57:3325-30.

28. Barber DL, Wherry EJ, Ahmed R. Cutting edge: rapid in vivo killing by memory CD8 T cells. J Immunol 2003;171:27-31.

29. Schwarz K, van den Broek M, Kostka S, Kraft R, Soza A, Schmidtke G, Kloetzel
PM, Groettrup M. Overexpression of the proteasome subunits LMP2, LMP7, and MECL-1 but not PA28 $\alpha / \beta$ enhances the presentation of an immunodominant lymphocytic choriomeningitis virus $\mathrm{T}$ cell epitope. J Immunol 2000;165:768-78.

30. Blomberg K, Hautala R, Lovgren J, Mukkala VM, Lindqvist C, Akerman K. Time-resolved fluorometric assay for natural killer activity using target cells labelled with a fluorescence enhancing ligand. J Immunol Methods 1996;193: 199-206.

31. Martin-Fonteccha A, Sebastiani S, Höpken UE, Uguccioni M, Lipp M, Lanzavecchia A, Sallusto F. Regulation of dendritic cell migration to the drining lymph node: impact on T lymphocyte traffic and priming. $J$ Exp Med 2003;198: 615-21.

32. Hsu FJ, Benike C, Fagnoni F, Liles TM, Czerwinski D, Taidi B, Engleman EG, Levy R. Vaccination of patients with B-cell lymphoma using autologous antigenpulsed dendritic cells. Nat Med 1996;2: 52-8.

33. Nestle FO, Alijagic S, Gilliet M, Sun Y, Grabbe S, Dummer R, Burg G, Schadendorf D. Vaccination of melanoma patients with peptide- or tumor lysatepulsed dendritic cells. Nat Med 1998;4: 328-32.

34. Schuler-Thurner B, Schultz ES, Berger TG, Weinlich G, Ebner S, Woerl P, Bender A, Feuerstein B, Fritsch PO, Romani N, Schuler G. Rapid induction of tumorspecific type $1 \mathrm{~T}$ helper cells in metastatic melanoma patients by vaccination with mature, cryopreserved, peptide-loaded monocyte-derived dendritic cells. J Exp Med 2002;195:1279-88.

35. Schuler G, SchulerThurner B, Steinman RM. The use of dendritic cells in cancer immunotherapy. Curr Opin Immunol 2003; 15:138-47.

36. Waeckerle-Men Y, Uetz-von Allmen E, Fopp M, von Moos R, Boehme C, Schmid HP, Ackermann D, Cerny T, Ludewig B, Groettrup M, Gillessen S. Dendritic cellbased multi-epitope immuntherapy of hormone-refractory prostate carcinoma. Cancer Immunol Immunother 2006;55: 1524-33.

37. Schultze V, D'Agosto V, Wack A, Novicki D, Zorn J, Hennig R. Safety of MF59 adjuvant. Vaccine 2008;26:3209-22.

38. Brewer JM. (How) do aluminium adjuvants work? Immunol Lett 2006;102: 10-15.

39. Wack A, Baudner BC, Hilbert AK, Manini I, Nuti S, Tavarini S, Scheffczik H, Ugozzoli M, Singh M, Kazzaz J, Montomoli E, Del Giudice G, et al. Combination adjuvants for the induction of potent, long-lasting antibody and T-cell 
responses to influenza vaccine in mice. Vaccine 2008;26:552-61.

40. Ott G, Barchfeld GL, Chernoff D, Radhakrishnan R, van Hoogevest P, Van Nest G. MF59. Design and evaluation of a safe and potent adjuvant for human vaccines. Pharm Biotechnol 1995;6:277-96.

41. Casella CR, Mitchell TC. Putting endotoxin to work for us: monophosphoryl lipid A as a safe and effective vaccine adjuvant. Cell Mol Life Sci 2008;65:3231-40.

42. Evans JT, Cluff CW, Johnson DA, Lacy MJ, Persing DH, Baldridge JR. Enhancement of antigen-specific immunity via the TLR4 ligands MPL adjuvant and Ribi.529. Expert Rev Vaccines 2003;2: 219-29.

43. Waite DC, Jacobson EW, Ennis FA, Edelman R, White B, Kammer R, Anderson C, Kensil CR. Three doubleblind, randomized trials evaluating the safety and tolerance of different formulations of the saponin adjuvant QS21. Vaccine 2001;19:3957-67.
44. Ballou WR. The development of the RTS,S malaria vaccine candidate: challenges and lessons. Parasite Immunol 2009;31: 492-500.

45. Didierlaurent AM, Morel S, Lockman L, Giannini SL, Bisteau M, Carlsen H, Kielland A, Vosters O, Vanderheyde $\mathrm{N}$, Schiavetti F, Larocque D, Van Mechelen $\mathrm{M}$, et al. ASO4, an aluminum salt- and TLR4 agonist-based adjuvant system, induces a transient localized innate immune response leading to enhanced adaptive immunity. J Immunol 2009;183: 6186-97.

46. Giannini SL, Hanon E, Moris P, Van Mechelen M, Morel S, Dessy F, Fourneau MA, Colau B, Suzich J, Losonksy G, Martin MT, Dubin G, et al. Enhanced humoral and memory B cellular immunity using HPV16/18 L1 VLP vaccine formulated with the MPL/aluminium salt combination (AS04) compared to aluminium salt only. Vaccine 2006;24: 5937-49.
47. Welters MJ, Kenter GG, Piersma SJ, Vloon AP, Lowik MJ, Berends-van der Meer DM, Drijfhout JW, Valentijn AR, Wafelman AR, Oostendorp J, Fleuren GJ, Offringa R, et al. Induction of tumor-specific $\mathrm{CD} 4^{+}$and $\mathrm{CD}^{+}{ }^{+} \mathrm{T}$-cell immunity in cervical cancer patients by a human papillomavirus type 16 E6 and E7 long peptides vaccine. Clin Cancer Res 2008;14:178-87.

48. Leffers N, Lambeck AJ, Gooden MJ, Hoogeboom BN, Wolf R, Hamming IE, Hepkema BG, Willemse PH, Molmans BH, Hollema H, Drijfhout JW, Sluiter WJ, et al. Immunization with a P53 synthetic long peptide vaccine induces P53-specific immune responses in ovarian cancer patients, a phase II trial. Int J Cancer 2009; 125:2104-13.

49. Men Y, Gander B, Merkle HP, Corradin G. Induction of sustained and elevated immune responses to weakly immunogenic synthetic malarial peptides by encapsulation in biodegradable polymer microspheres. Vaccine 1996;14:1442-50. 